\title{
Vitiligo and chronic autoimmune thyroiditis
}

\author{
Florica Sandru ${ }^{1,2}$, Mara Carsote ${ }^{3,4 *}$, Simona Elena Albu ${ }^{5,6}$, Mihai Cristian Dumitrascu ${ }^{5,6}$, Ana Valea ${ }^{7,8}$
}

\section{Author Affiliations:}

1. Department of Dermatology, Elias Emergency University Hospital, Bucharest, Romania

2. Department of Dermatology, Carol Davila University of Medicine and Pharmacy, Bucharest, Romania

3. Department of Endocrinology, G. I. Parhon National Institute of Endocrinology, Bucharest, Romania

4. Department of Endocrinology, Carol Davila University of Medicine and Pharmacy, Bucharest, Romania

5. Department of Obstetrics and Gynecology, Emergency University Hospital, Bucharest, Romania

6. Department of Obstetrics and Gynecology, Carol Davila University of Medicine and Pharmacy, Bucharest, Romania

7. Department of Endocrinology, Clinical County Hospital, Cluj-Napoca, Romania

8. Department of Endocrinology, Iuliu Hatieganu University of Medicine and Pharmacy, Cluj-Napoca, Romania

\author{
* Corresponding Author: \\ Mara Carsote, \\ C.I.Parhon National Institute of \\ Endocrinology, Aviatorilor Ave \\ 34-38, 011683, \\ Bucharest, Romania. \\ E-mail: carsote_m@hotmail.com
}

DOI

10.25122/jml-2019-0134

\section{Dates}

Received: 11 December 2019

Accepted: 23 March 2021

\section{ABSTRACT}

Vitiligo, the discoloration of the skin, has different autoimmune mechanisms reflected by many biomarkers as shown by skin histology, staining for CD4 and CD8 T lymphocytes, chemokine ligand 9 or circulating cytokines such as interleukin (IL)-1 beta, interferon (IFN)-gamma, transforming growth factor (TGF)-beta, antibodies, markers of oxidative stress, chemokines, and others. In this narrative review, we aim to overview vitiligo in relationship with chronic autoimmune thyroiditis. Regarding vitiligo, more than 50 different genetic loci have been associated with this disease, and the heritability is high. There is a $20 \%$ risk of an environmental connection which may also act as a trigger; moreover, the association with human leukocyte antigen (HLA) expression is well recognized. The specific lesions display CD8+ tissue-resident memory $\mathrm{T}$ cells as continuous key activators of melanocytes. The association with chronic thyroiditis is based on common autoimmune background and excessive reactive oxygen species that destroy melanocytes and thyrocytes (oxidative stress hypothesis) with thyroxine and melanin as target molecules, thus sharing a common origin: tyrosine. Moreover, common epigenetic anomalies or mutations of the Forkhead transcription factor D3 (FOXD3) have been described. Since vitiligo affects up to $1-2 \%$ of the population worldwide and $34 \%$ of patients have positive thyroid antibodies, apart from common autoimmunity background and oxidative stress toxicity, the association is clinically relevant for different practitioners.

KEYWORDS: vitiligo, thyroiditis, antibodies.

ABBREVIATIONS: ATG - anti-thyroglobulin antibodies; FOXD3 - forkhead transcription factor D3; IFN - interferon; IL - interleukin; JAK-STAT - Janus kinase-signal transducer and activator of transcription; ROS - reactive oxygen species; TGF - transforming growth factor; TPO - thyroid peroxidase antibodies. 


\section{JOURNAL of MEDICINE and LIFE}

\section{INTRODUCTION}

Vitiligo represents the discoloration of the skin, a condition with rather limited therapeutical options nowadays [1]. Regarding the etiology, the autoimmune component is well known, but the exact mechanisms are still a matter of debate [2,3]. The mechanisms of this condition are reflected by biomarkers like skin histology, including additional staining for CD4 and CD8 T lymphocytes on the one hand, or markers like chemokine ligand 9, different circulating cytokines: interleukin (IL)-1 beta, interferon (IFN)-gamma, transforming growth factor (TGF)-beta, antibodies, markers of oxidative stress, chemokines on the other hand [2, 3].

Another example is IL-17, which is connected with different autoimmunity conditions, and its level is high in vitiligo; in addition to the lesion's clinical aspects, a positive correlation with disease activity has been described [2, 3]. Moreover, IL-17 may be modulated by ultraviolet B phototherapy, which is used for vitiligo $[2,3]$. IL-17 has been linked with other chronic skin inflammation like psoriasis, acne, atopic dermatitis, and autoimmune-derived diseases like alopecia areata [4]. Poor prognosis factors are clinically described as Koebner's phenomenon, confetti-like lesions, and poorly defined borders [5].

Since vitiligo impacts the quality of life, different therapies are under consideration, and phototherapy remains the first-line approach which represents an option for different dermatoses as well [6-8]. Early recognition and prompt therapy seem to improve the prognosis [9]. The efficacy of other alternatives like platelet-rich plasma is still insufficiently documented [10]. In this paper, we aimed to overview vitiligo in relationship with chronic autoimmune Hashimoto's thyroiditis.

\section{MATERIAL AND METHODS}

We conducted a narrative review based mainly but not exclusively on PubMed database research. A number of 50 references were found and are described in detail in this paper.

\section{RESULTS}

\section{Vitiligo}

The melanocytes' progressive autoimmune deterioration causes lesions at the level of skin and hair [11]. The process is linked to both genetic and environmental factors, and multiple aspects need to be taken into consideration when it comes to discussing the complex pathogeny of the condition.

More than 50 genes carry the disease susceptibility. The heritability percent is increased, and there is a $20 \%$ risk of an environmental connection that may also act as a trigger in some cases. [11] The association with human leukocyte antigen (HLA) expression is well recognized [1 1]. Immune anomalies are underlining vitiligo and also connecting it with the risk of developing melanoma [12]. Signal transduction pathways like the Janus kinase-signal transducer and activator of transcription (JAK-STAT) play a role in the pathogenic loop of vitiligo [13]. Drugs targeting the JAK-STAT pathway are encouraging, seem safe, and efficient in re-pigmentation but still irrelevant in daily practice on a larger scale [14, 15].

Apart from non-receptor tyrosine kinases pathways like JAK-STAT, signals from the tyrosine kinases network also seem to contribute to the autoimmune-related background involving the skin acquired condition [16]. Also, the lesions of vitiligo display CD8+ resident memory $\mathrm{T}$ cells as continuous key activators of the destruction of pigment-producing cells [17]. Another mechanism is related to the excessive local production of reactive oxygen species (ROS) in melanocytes about to be destroyed [18].

\section{Chronic autoimmune thyroiditis}

The autoimmune Hashimoto's thyroiditis has a relatively high frequency in the general population with a female predisposition and potential positive family history of autoimmune conditions [19,20]. It is regarded as the most common autoimmune disease and the most important cause of hypothyroidism in iodine non-deficient areas. It is also the most common cause of hypothyroidism in children and teenagers nowadays $[19,20]$. The clinical presentation may be irrelevant or autoimmune hypothyroidism (including the severe pattern of myxedema) may be detected [19, 20]. The presence of other endocrine and non-endocrine conditions may actually be the reason for admission $[19,20]$. The thyroid gland could have a normal volume or be enlarged, typically without nodules [19, 20]. It underlines specific hypoechoic ultrasound features [19, 20]. Atrophic autoimmune myxedema has also been described, especially in the elderly, who usually have a reduced gland diameter $[19,20]$.

Thyroid antibodies that are causing the condition are well known and easily tested in everyday practice: thyroid peroxidase antibodies (TPO) and anti-thyroglobulin antibodies (ATG) [21, 22]. Increased antibody levels seen a least once on a blood test is enough to establish the diagnosis in daily practice [21,22]. Their levels may be fluctuating in different patterns. Nonetheless, the most relevant for 


\section{JOURNAL of MEDICINE and LIFE}

routine assessments are TPO [21, 22]. Genetic background causing the autoimmunity activation together with environmental factors including nutrients (like selenium deficiency) have been described as parts of the pathogen network [22-24].

Thyroid function may be normal in chronic autoimmune thyroiditis, but only for a while; in time, subclinical/clinical hypothyroidism develops due to the blocking effect of the antibodies on the thyroid [24, 25]. Transitory thyrotoxicosis can be rarely found [24, 25]. The pathological report shows lymphocytic infiltration of the thyroid and follicular cells may become atrophic [26]. Thyroid ultrasound remains the most helpful tool for diagnosis, except for antibody values [27]. Low thyroid function requires levothyroxine substitution, while thyroidectomy is rarely indicated unless local compressive symptoms are present [28].

However, 20\% of patients associate at least one more autoimmune disease [29-31]. Regarding the malignancy risk, the condition has been correlated with papillary thyroid cancer, increasing the risk of primary thyroid lymphoma [29-31]. Due to the high prevalence in the general population, the overlap with other frequently found conditions like obesity, metabolic syndrome, or breast cancer has been analyzed, and the association is still a matter of debate [32-34].

\section{Vitiligo and chronic autoimmune thyroiditis}

Vitiligo and Hashimoto's thyroiditis represent two well-known autoimmune diseases, noting that vitiligo is the most common condition which involves skin depigmentation, found in up to $1-2 \%$ of the population [35]. The autoimmune background is common between the diseases; thus, the association involves more than antibodies [35]. Because of the incidental co-presence of the two conditions, thyroid antibodies are encouraged to be routinely tested in patients with vitiligo [36]. On the other hand, vitiligo is part of the non-endocrine conditions included in poly-glandular autoimmune syndrome as well as gastritis and hepatitis of autoimmune cause, celiac disease, pernicious anemia, and others [37]. The reported prevalence of positive thyroid antibodies is $34 \%$, with different results depending on ethnicity [38, 39].

Another common hypothesis of the two disorders includes reactive oxygen species in high amounts that trigger conditions, destroying melanocytes and thyrocytes (oxidative stress hypothesis) [40,41]. The target molecules thyroxine and melanin share a common origin: tyrosine [40,41]. A new therapeutic approach is under consideration using quinone derivatives [40]. Their role is to combat oxidative stress-related autoimmunity [40].

Also, common epigenetic anomalies have been previously described [42]. Mutations of the Forkhead transcription factor D3 (FOXD3) are linked to positive thyroid antibodies and the diagnosis of vitiligo [43]. Clinical presentation varies; one prevalence study identified positive antibodies in patients with vitiligo but with an asymptomatic thyroid condition [44]. The association is also found in young patients [45].

\section{DISCUSSION}

When it comes to vitiligo and non-thyroid autoimmune conditions, some studies pointed to a potential common association with diabetes mellitus. [46]. This may be or not a part of a polyglandular autoimmune syndrome. We should also mention premature ovarian failure (the ovarian function stops before the age of 40), which includes the fact that one-third of females have autoimmune morbidities, including vitiligo and Hashimoto's thyroiditis [47].

The most frequent is chronic thyroiditis, but vitiligo has also been described with a higher risk than the general population [47]. A sex-chromosomal disorder that increases the risk of any autoimmune disease is Turner syndrome [48, 49]. The syndrome includes a higher prevalence of autoimmune conditions, which are seen twice as much compared to normal women, and these disorders involve vitiligo, chronic thyroiditis, type 1 diabetes mellitus, celiac disease, and alopecia areata $[48,49]$. In this particular situation, the contributor to increased autoimmunity is the iXq chromosome and exposure to estradiol (due to iatrogenic complications) [48, 49]. Another genetic disease with a higher risk for both conditions, vitiligo and Hashimoto's thyroiditis, is Down syndrome, potentially with a more severe phenotype for the thyroid condition [50].

\section{CONCLUSION}

Since vitiligo affects up to $1-2 \%$ of the population worldwide and $34 \%$ of them have positive thyroid antibodies, apart from common autoimmunity background and oxidative stress toxicity, this association is clinically relevant for practitioners.

\section{ACKNOWLEDGMENTS}

\section{Conflict of interest}

The authors declare that there is no conflict of interest. 


\section{JOURNAL of MEDICINE and LIFE}

\section{REFERENCES}

1. Nordlund JJ. The Medical Treatment of Vitiligo: An Historical Review. Dermatol Clin. 2017 Apr;35(2):107-116.

2. Singh RK, Lee KM, Vujkovic-Cvijin I, Ucmak D, Farahnik B, Abrouk M, Nakamura M, Zhu TH7, Bhutani T, Wei M, Liao W. The role of IL-17 in vitiligo: A review. Autoimmun Rev. 2016 Apr;15(4):397-404.

3. Speeckaert R, Speeckaert M, De Schepper S, van Geel N. Biomarkers of disease activity in vitiligo: A systematic review. Autoimmun Rev. 2017 Sep;16(9):937-945.

4. Bernardini N, Skroza N, Tolino E, Mambrin A, Anzalone A, Balduzzi V, Colapietra D, Marchesiello A, Michelini S, Proietti I, Potenza C. IL-17 and its role in inflammatory, autoimmune, and oncological skin diseases: state of art. Int J Dermatol. 2019 Oct 30. doi: 10.1111/iji.14695. [T ahead of print].

5. van Geel N, Grine L, De Wispelaere P, Mertens D2, Prinsen CAC, Speeckaert R. Clinical visible signs of disease activity in vitiligo: a systematic review and meta-analysis. J Eur Acad Dermatol Venereol. 2019 Sep;33(9):1667-1675.

6. Zubair R, Hamzavi IH. Phototherapy for Vitiligo. Dermatol Clin. 2020 Jan;38(1):55-62.

7. Jacob J, Pona A, Cline A, Feldman S. Home UV Phototherapy. Dermatol Clin. 2020 Jan;38(1):109-126.

8. Chang HC, Lin MH, Tsai HH. Efficacy of Combination Therapy With Fractional Carbon Dioxide Laser and Ultraviolet B Phototherapy for Vitiligo: A Systematic Review and Meta-Analysis. Aesthet Surg J. 2019 Oct 12. pii: sjz250. doi: 10.1093/asj/sjz250. [Epub ahead of print]

9. Nahhas AF, Braunberger TL, Hamzavi IH. Update on the Management of Vitiligo. Skin Therapy Lett. 2019 May;24(3):1-6.

10. Hesseler MJ, Shyam N. Platelet-rich plasma and its utility in medical dermatology: A systematic review. J Am Acad Dermatol. 2019 Sep;81(3):834-846.

11. Roberts GHL, Santorico SA, Spritz RA. The genetic architecture of vitiligo. Pirment Cell Melanoma Res. 2019 Nov 19. doi: 10.1111/pcmr. 12848. [Epub ahead of print].

12. Failla CM, Carbone ML, Fortes C, Pagnanelli G, D'Atri S. Melanoma and Vitiligo: In Good Company. Int J Mol Sci. 2019 Nov 15;20(22). pii: E5731. doi: 10.3390/ijms20225731.

13. Howell MD, Kuo FI, Smith PA. Targeting the Janus Kinase Family in Autoimmune Skin Diseases. Front Immunol. 2019 Oct 9;10:2342. doi: 10.3389/fimmu.2019.02342. eCollection 2019

14. Montilla AM, Gómez-García F, Gómez-Arias PJ, Gay-Mimbrera J, Hernández-Parada J, Isla-Tejera B, Ruano J. Scoping Review on the Use of Drugs Targeting JAK/STAT Pathway in Atopic Dermatitis, Vitiligo, and Alopecia Areata. Dermatol Ther (Heidelb). 2019 Dec;9(4):655-683.

15. Relke N, Gooderham M. The Use of Janus Kinase Inhibitors in Vitiligo: A Review of the Literature. J Cutan Med Surg. 2019 May/Jun;23(3):298-306

16. Szilveszter KP, Németh T, Mócsai A. Tyrosine Kinases in Autoimmune and Inflammatory Skin Diseases. Front Immunol. 2019 Aug 9;10:1862. doi: 10.3389/fimmu.2019.01862. eCollection 2019

17. Riding RL, Harris JE. The Role of Memory CD8+ T Cells in Vitiligo. J Immunol. 2019 Jul 1;203(1):11-19.

18. Wang Y, Li S, Li C. Perspectives of New Advances in the Pathogenesis of Vitiligo: From Oxidative Stress to Autoimmunity. Med Sci Monit. 2019 Feb 6;25:1017-1023

19. Caturegli P, De Remigis A, Rose NR. Hashimoto thyroiditis: clinical and diagnostic criteria. Autoimmun Rev 2014 Apr-May;13(4-5):391-7.

20. Liontiris MI, Mazokopakis EE. A concise review of Hashimoto thyroiditis (HT) and the importance of iodine, selenium, vitamin $\mathrm{D}$ and gluten on the autoimmunity and dietary management of HT patients.Points that need more investigation. Hell J Nucl Med. 2017 Jan-Apr;20(1):51-56.

21. Ragusa F, Fallahi P, Elia G, Gonnella D, Paparo SR, Giusti C, Churilov LP, Ferrari SM, Antonelli A. Hashimotos thyroiditis: Epidemiology, pathogenesis, clinic and therapy. Best Pract Res Clin Endocrinol Metab. 2019 Nov 26:101367. doi: 10.1016/j.beem.2019.101367. [Epub ahead of print].

22. Rayman MP. Multiple nutritional factors and thyroid disease, with particular reference to autoimmune thyroid disease. Proc Nutr Soc. 2019 Feb;78(1):34-44.

23. Richard-Eaglin A, Smallheer BA. Immunosuppressive/Autoimmune Disorders. Nurs Clin North Am. 2018 Sep;53(3):319-334.

24. Merrill SJ, Minucci SB. Thyroid Autoimmunity: An Interplay of Factors. Vitam Horm. 2018;106:129-145.

25. Biondi B, Cappola AR, Cooper DS. Subclinical Hypothyroidism: A Review. JAMA. 2019 Jul 9;322(2):153-160.

26. Bliddal S, Nielsen CH, Feldt-Rasmussen U. Recen advances in understanding autoimmune thyroid disease: the tallest tree in the forest of polyautoimmunity. F1000Res. 2017 Sep 28;6:1776.

27. Wu G, Zou D, Cai H, Liu Y. Ultrasonography in the diagnosis of Hashimoto's thyroiditis. Front Biosci (Landmark Ed). 2016 Jun 1;21:1006-12.

28. Gan T, Randle RW. The Role of Surgery in Autoimmune Conditions of the Thyroid. Surg Clin North Am. 2019 Aug;99(4):633-648.

29. Mancuso S, Carlisi M, Napolitano M, Siragusa S. Lymphomas and thyroid: Bridging the gap. Hematol Oncol. 2018 Feb 27. doi: 10.1002/hon.2504. [Epub ahead of print]

30. Valea A, Albu SE, Petrescu R, Carsote M. Chronic thyroiditis and differentiated thyroid cancer: from overlapping high prevalence to a shift on multifocal intra-thyroid papillary thyroid carcinoma. Journal of Surgical Sciences. 2017:4(1):38-45.

31. Boi F, Pani F, Mariotti S. Thyroid Autoimmunity and Thyroid Cancer: Review Focused on Cytological Studies. Eur Thyroid J. 2017 Jul;6(4):178-186.

32. Valea A, Carsote M, Moldovan C, Georgescu C. Chronic autoimmune thyroiditis and obesity. Archives of the Balkan Medical Union. 2018;53(1):64-69.

33. Valea A, Carsote M, Morar A, Mihalca D, Tarau R, Marcusanu A, Talpasanu A, Chereji A, Georgescu CE. High cardio-metabolic risk parameters: a cross-sectional study in 80 women and men diagnosed with chronic thyroiditis (an age decades analysis). Current Health Sciences Journal. 2015.41(Suppl.1):101-104.

34. Valea A, Ghervan C, Morar A, Pop DD, Carsote M, Albu SE, Georgescu CE, Chiorean A. Hashimoto's thyroiditis and breast cancer: coincidence or correlation?. Archives of Balkan Medical Union. 2016;51(1):129-132.

35. Baldini E, Odorisio T, Sorrenti S, Catania A, Tartaglia F, Carbotta G, Pironi D, Rendina R, D'Armiento E, Persechino S, Ulisse S. Vitiligo and Autoimmune Thyroid Disorders. S, Ulisse S. Vitiligo and Autoimmune Thyroid Disont Endocrinol (Lausanne). 2017 Oct 27;8:290. Front Endocrinol (Lausanne). 2017 Oct 27;8:290.
doi: 10.3389/fendo.2017.00290. eCollection 2017.

36. Liu M, Murphy E, Amerson EH. Rethinking screening for thyroid autoimmunity in vitiligo. J Am Acad Dermatol. 2016 Dec;75(6): 1278-1280

37. Wémeau JL, Proust-Lemoine E, Ryndak A, Vanhove L. Thyroid autoimmunity and polyglandular endocrine syndromes. Hormones (Athens). 2013 Jan-Mar;12(1):39-45.
38. Vachiramon V, Harnchoowong S, Onprasert W, Chanprapaph K. Prevalence of Thyroid Abnormalitie in Thai Patients with Vitiligo. Biomed Res Int. 2017;2017:7502935. doi: 10.1155/2017/7502935.

39. Díaz-Angulo S, López-Hoyos M, Muñoz-Cacho P, López-Escobar M, González-López MA. High prevalence of thyroid autoimmunity in patients with alopecia areata and vitiligo: a controlled study. Australas J Dermatol. 2015 May;56(2):142-3.

40. Li D, Liang G, Calderone R, BellantiJA. Vitiligo and Hashimoto's thyroiditis: Autoimmune diseases linked by clinical presentation, biochemical commonality, and autoimmune/oxidative stress-mediated toxicity pathogenesis. Med Hypotheses. 2019 Jul; 128:69-75.

41. Baldini E, Odorisio T, Tuccilli C, Persechino S, Sorrent S, Catania A, Pironi D, Carbotta G, Giacomelli L, Arcieri $\mathrm{S}$, Vergine M, Monti M, Ulisse S. Thyroid diseases and skin autoimmunity. Rev Endocr Metab Disord. 2018 Dec;19(4):311-323.

42. Schunter JA, Löffler D, Wiesner T, Kovacs P, Badenhoop K, Aust G, Tönjes A, Müller P, Baber R, Simon JC, Führer D, Pfäfle RW, Thiery J, Stumvoll M, Kiess W, Kratzsch J, Körner A. A novel FoxD3 Variant Is Associated With Vitiligo and Elevated Thyroid Auto-Antibodies. J Clin Endocrinol Metab. 2015 Oct;100(10):E1335-42.

43. Colucci R, Dragoni F, Moretti S. Oxidative stress and Cell Longev. 2015;2015:631927. doi: 10.1155/2015/631927.

44. Dash R, Mohapatra A, Manjunathswamy BS. Anti-thyroid peroxidase antibody in vitiligo: prevalence study. J Thyroid Res. 2015;2015:192736. doi prevalence study. J Thy

45. Yang Y, Huang G, Yan X, Oing Z, Clinical Analysis of Thyroglobulin Antibody and Thyroid Peroxidase Antibody and their Association with Vitiligo. Indian J Dermatol. 2014 Jul; 59(4):357-60.

46. Chang HC, Lin MH, Huang YC, Hou TY. The association between vitiligo and diabetes mellitus: A systematic review and meta-analysis. J Am Acad Dermatol. 2019 Dec;81(6):1442-1445.

47. Ayesha, Jha V, Goswami D. Premature Ovarian Failure: An Association with Autoimmune Diseases. J Clin Diagn Res. 2016 Oct;10(10):OC10-OC12.

48. De Sanctis V, Khater D. Autoimmune diseases in Turne syndrome: an overview. Acta Biomed. 2019 Sep 6;90(3):341-344.

49. Wegiel M, Antosz A, Gieburowska J, Szeliga K, Hankus M, Grzybowska-Chlebowczyk U, Wiecek S, Malecka-Tender E, Gawlik A. Autoimmunity Predisposition in Girls With E, Gawlik A. Autoimmunity Predisposition in Girls With
Turner Syndrome. Front Endocrinol (Lausanne). 2019 Jul 30;10:511. doi: 10.3389/fendo.2019.00511. eCollection 2019.

50. Aversa T, Crisafulli G, Zirilli G, De Luca F, Gallizzi $\mathrm{R}$, Valenzise M. Epidemiological and clinical aspects of autoimmune thyroid diseases in children with Down's syndrome. Ital J Pediatr. 2018 Mar 21;44(1):39. doi: 10.1186/s13052-018-0478-9. 